\title{
Guillaume Budé à son médecin: un inédit sur sa maladie
}

\author{
GUYLAVOIE
}

$\mathrm{C}$ 'est, semble-t-il, dans le Catalogue 1 de l'exposition sur Guillaume Budé tenue à la Bibliothèque nationale en 1968 qu'est signalée pour la première fois l'existence de la copie d'une lettre du célèbre humaniste à son médecin et ami Guillaume $\mathrm{Cop}^{2}$. Cette lettre compte parmi les plus intéressantes de l'humaniste français et il vaut la peine, après une description de la copie, d'en faire une brève analyse avant d'en présenter la traduction.

La copie retrouvée figure dans l'exemplaire personnel de Budé de son $D e$ Philologia publié en 1532. A la fin du volume, en dessous du privilège royal imprimé sur les deux tiers de la dernière page, commence la lettre qui se poursuit sur les trois pages blanches suivantes pour se terminer sur la garde. Après quarante-cinq lignes en latin, entrecoupées de quelques mots grecs, Budé utilise le grec jusqu'à une conclusion de vingt lignes où grec et latin alternent à parts égales. Attribuable à deux mains inconnues, dont l'une est plus soignée que l'autre, la copie est parsemée de ratures-plus de deux cent cinquante—et retient quelques fautes suggérant que les copistes n'ont pas bien compris le texte grec. En outre, les lignes 16 et 17 de la dernière page souffrent d'une lacune ou d'une erreur de transcription qui les rend incompréhensibles. De la date de la copie, la seule chose dont nous puissions être certains est qu'elle est postérieure à 1532, année de la publication du De Philologia.

La lettre elle-même n'est pas datée, mais on peut connaître approximativement le moment où elle a été écrite par le détail suivant que fournit Budé: "il y a déjà presque sept ans que je souffre de ce mal"3. Or, le 14 mars 1510, Budé avait noté dans une lettre à Lascaris: "voilà déjà cinq pleines années que je suis affligé d'une mauvaise santé presque continuelle"4. Indication confirmée dans la lettre à Erasme du 7 juillet 1516: “... cette funeste maladie qui, depuis onze ans, m'a embarrassé de tous les ennuis possibles"5. De Même, le 2 février 1520 il écrit à Louis Vivès: “J'ai aussi été malade depuis le début de l'hiver, ce qui, depuis 15 ans, m'arrive à peu près tous les deux ans" $"$. Le début de la maladie de Budé 
prend donc place en $1505^{7}$. On peut ainsi situer la lettre à Cop en 1512 , puisque Budé est malade depuis sept ans au moment où il l'écrit.

Cette date explique que la lettre ne figure ni dans les Epistolae de 1520, ni dans les Epistolae Posteriores de 1522. Le 10 janvier 1521, Budé explique en effet à Louis Vivès que son premier recueil contient seulement des lettres écrites au cours des deux années précédant la parution et il note: “je ne garde pas de copie des lettres que j'envoie à mes amis les plus intimes" 8 . Celle qu'il avait adressée à Cop compte pourtant parmi ces textes soignés, parmi ces morceaux d'apparat dont les humanistes étaient fiers et souhaitaient la circulation, comme le suggère la remarque de Budé vers la fin: "Je serais certes digne de votre indulgence, à toi et aux autres savants qui verront cette lettre" .

Par son allure générale, la lettre à Guillaume Cop est déjà ce que seront les longues missives adressées quelques années plus tard à son frère Louis, à Christophe de Longueil ou à Tunstall ${ }^{10}$. Encore en 1529, l'épître "aux jeunes hellénistes" qui termine les Commentarii linguae graecae présentera des ressemblances frappantes avec elle ${ }^{11}$. Le vocabulaire comporte nombre de mots fort rares comme $\mu \alpha{ }^{\prime} \mu \alpha \alpha$, ou encore des mots forgés, comme

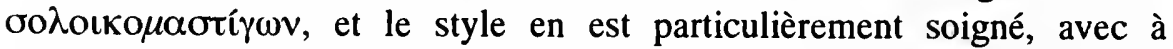
l'occasion d'interminables périodes. Mais, surtout, on y retrouve déjà nombre de thèmes qui s'avéreront des constantes sous la plume de Budé.

Ecrivant à son médecin, celui-ci traite naturellement longuement de cette maladie sur laquelle il reviendra sans cesse. Tout comme reviendront continuellement sa passion pour la philologie, son dévouement pour la cause des lettrés, l'indulgence qu'il sollicite de leur part, l'inquiétude de posséder une maîtrise insatisfaisante de la langue grecque, la comparaison avec les compétitions du stade où il ne peut manquer d'être vaincu, le rôle joué par l'étude dans sa maladie, l'impossibilité de tirer de la pratique des lettres un revenu convenable, son indignation contre les adeptes d'une langue négligée, ses plaintes contre l'aveuglement injuste du sort, la soumission à la Providence, l'incapacité où il est de contenir sa plume une fois lancée. Par rapport à la correspondance publiée en 1520 et 1522, cette lettre fait presque figure de programme en ce qui touche les thèmes d'ordre général.

Bien qu'elle soit omniprésente dans l'oeuvre de Budé, la maladie y plane en quelque sorte comme une ombre aussi insaisissable que menaçante. On sait que tout son entourage s'en inquiétait vivement, aussi bien ses proches que son épouse, sans compter les médecins qui le menaçaient de mort ${ }^{12}$. Mais, contrairement à son inséparable pendant hollandais, Budé ne donne jamais d'indices sur la nature de son mal ${ }^{13}$. Pourtant, il est certain que Budé s'intéressait vivement à la médecine et en possédait des connaissances 
sérieuses. Son père, Jean Budé, "grand acheteur de livres"14, qui approchait les soixante-dix ans au moment où Budé se mit à l'étude, “avait été malade sans répit depuis l'âge de cinquante ans et, partant, avait étudié la médecine et s'y connaissait"15. Sur quarante et un manuscrits retracés par H. Omont comme ayant appartenu à Jean et à Guillaume Budé, neuf sont d'ordre médical ${ }^{16}$. Relevons aussi la lettre du 14 mars 1510 à Jean Lascaris, où Budé écrit: 'J'escompterais grandement, si tu jouissais d'un loisir un peu suivi, que tu voies à me faire copier quelques livres de Galien (...). De nombreux titres, je voudrais surtout une copie des suivants:De differentia morborum, De optima corporis nostri constitutione, De elementis secundum Hippocratem, De facultatibus naturalibus, Ars Parva." Et plus loin: "la lecture de la Thérapeutique m'a intéressé aux théories de cet auteur"17. On sait aussi que Budé tenait en haute estime Linacre qu'il avait connu à Paris et dont il avait lu des traductions de Galien ${ }^{18}$. En outre, parmi ses amis parisiens, Budé compte non seulement le médecin Guillaume Cop, mais aussi Jean Ruel, "étroitement associé à mes études", dira-t-il ${ }^{19}$.

Dans ce contexte, le silence de Budé sur la nature des maux dont il souffrait peut étonner et la lettre à Guillaume Cop occupe une place particulière, puisque c'est le seul endroit où Budé fournit des précisions sur les maux qui l'accablaient ${ }^{20}$. A la page deux de la copie, les lignes 23 à 25 rappellent que la maladie remonte à sept ans. Ce rappel ne s'explique vraiment que si l'accès décrit est la manifestation habituelle du mal. Il faut toutefois se demander si la description que Budé fait de ses maux à son ami est compatible avec les symptômes relevés par Louis Le Roy dans sa Vita Budaei, parue en 1540, année même de la mort du grand humaniste, et seul autre endroit où l'on trouve des détails sur cette maladie. Malgré sa longueur, ce texte vaut d'être cité. En voici la traduction:

"Quand son amour de l'étude finit par l'emporter sur son attachement à la vie, quand la découverte de ce bien l'amena à n'accorder aucun prix à l'existence, il fut atteint d'une longue et grave maladie dont il fut affligé pendant plus de vingt ans $^{21}$, au point que toute gaieté disparut de son visage, toute jovialité disparut de son âme, tout sourire disparut dans son accueil, toute urbanité et toute affabilité dans son commerce, au point aussi que son amour des lettres de jour en jour plus grand en était brimé. Si bien qu'il n'était même plus le reflet, l'ombre de lui-même, mais présentait l'aspect d'un mort vivant. Voici comment se présentait sa maladie. Sa gorge, qui enflait, lui causait une terrible douleur et il se mettait à tousser bruyamment ${ }^{22}$. Il en éprouvait la nuit une telle frayeur que le matin il s'étonnait d'être indemne en pensant à sa respiration bloquée et soudain libérée. Bien au fait, son épouse pressentait l'arrivée des crises et soulageait le mal de son mari par de fréquents changements de position et par des tapes 
aux épaules. De cette maladie venait une grande pâleur, la chute des cheveux, la maigreur et l'engourdissement de tout le corps, une extrême faiblesse de tous les membres. Les médecins, à qui il l'avait maintes fois exposée, ne pouvaient croire à un si grand mal dont ils ne retraçaient aucun exemple. Les crises survenaient à peu près tous les deux mois. Il souffrait en outre d'un mal de tête incessant, qui le tourmentait terriblement quand il lisait ou rédigeait. Comme on attribuait cette maladie aux humeurs qui lui alourdissaient la tête, les médecins lui promettaient un grand soulagement de son mal si celles-ci trouvaient au sommet de la tête une issue par où s'échapper. Ils lui conseillèrent donc de se prêter à ce qu'on ouvrit au fer rouge une voie à travers l'épaisse enveloppe du dessus de la tête. Le crâne, disaient-ils, possède des ouvertures qui permettent aux vapeurs de s'échapper vers le haut. On procéda selon leur recommandation, ce qui fut un grand supplice ${ }^{23}$. Mais aucun soulagement ne résulta d'un traitement aussi risqué et pénible. En effet, les vapeurs denses et lourdes montaient au cerveau et s'y accumulaient, faute d'un passage vers le haut. En même temps, elles s'y épaississaient, puis se liquéfiaient pour être entraînées par leur propre poids dans le front, les tempes et les yeux, de sorte que la somnolence le gagnait sur son livre et que les plus grands efforts ne pouvaient l'empêcher de s'endormir ${ }^{24}$.

La description de Budé et celle de Louis Le Roy ont toute l'apparence de se compléter. Si l'on met ensemble les deux textes, on peut comprendre que Budé a souffert d'un mal chronique de l'âge de trente-sept ans jusqu'à, semble-t-il, l'âge de 61 ans environ ${ }^{25}$. Un violent mal de tête était installé pour ainsi dire en permanence. La pâleur et la maigreur de Budé se remarquaient. Les crises commençaient par une vive douleur à la gorge, avec enflure et difficulté à respirer. S'ensuivait une forte toux qui se terminait par des expectorations purulentes amenant un brusque soulagement. Budé éprouvait alors des nausées “à en défaillir". Il était ensuite vaincu par une insurmontable somnolence pendant quelques jours. Enfin, se manifestait une douleur qui frappait tantôt les yeux, tantôt les gencives, tantôt le ventre et tantôt "toutes les parties à la fois". La seule divergence porte sur la fréquence des crises. Tandis que pour Le Roy elles surviennent "à peu près tous les deux mois", Budé, dans la lettre à Vivès du 2 février 1520, parle d'une maladie qui revient “à peu près tous les deux ans". Peut-être la contradiction est-elle plus apparente que réelle. De l'ensemble des lettres de Budé, en effet, on croit comprendre que, dans sa maladie, il connaissait régulièrement des accès particulièrement graves. Il n'est pas impossible que la remarque de Le Roy rappelle ces accès.

Si Budé se tait sur la nature de son mal, il nous éclaire abondamment sur les circonstances où il se manifeste. Les médecins, dit-il, établissent un lien 
étroit entre l'activité intellectuelle et son mal: "La pratique de la philologie en était alors tenue responsable par les médecins..." "26. De même, il écrit à Longueil: "Je passe sous silence les fréquentes interruptions de mon travail dues aux médecins qui me menaçaient de trépas si je ne modifiais incontinent mon régime de vie et ne renonçais pas aux livres" ${ }^{27}$. L'avis des médecins est partagé par son entourage: "Et, répètent proches et parents, (...) je me meurs misérablement pour elle (la philologie). En effet, comme ils disent, depuis que je l'ai introduite chez moi, je n'ai pas l'apparence d'avoir pris de sages décisions, ni pour ma fortune ni pour ma santé. (...) Dans la lassitude engendrée par une maladie si longue et si pénible, qui n'aurait pas cédé aux ordonnances des médecins, aux supplications de sa femme ou aux conseils de ses amis, comme s'il avait fait son deuil de ses espérances littéraires?"28.

Il semblerait que l'intensité des crises allait de pair avec l'intensité du travail intellectuel. Quand, en 1508, il rédigea ses premières Annotations aux Pandectes "en plus ou moins sept mois" 29 , il entend leur donner une suite "si seulement me le permettent ma disponibilité et une santé qui me contraint non seulement à interrompre l'oeuvre commencée, mais même à y renoncer, à l'abandonner"30. De même, le travail gigantesque que constituent les Commentaires de la langue grecque a produit des effets désastreux sur sa santé: “Je tendais plus loin”, écrit-il dans la postface, "lorsque le mal m'a frappé avec une telle violence que, si je ne m'étais arrêté aussitôt, le destin m'aurait emporté"31. Par contre Budé est manifestement en bonne santé quand il s'occupe de l'aménagement de sa propriété de Saint-Maur ${ }^{32}$ et, sauf erreur, ne connaît jamais de manifestation de ce mal quand il se livre à des activités autres que l'étude. Même que, lors de sa participation à l'ambassade qui devait réunir à Montpellier les ambassadeurs de François $l^{\text {er }}$ et de Charles Quint, il écrit à Guillaume du Maine: "J'ai pris la décision (...) de ramener à la pratique des lettres mon esprit depuis longtemps déjà absent de son poste (...). Mais, pauvre moi, je crains qu'elle ne me cause encore des tracas, cette vieille maladie"33. C'est dire assez clairement qu'il se porte bien quand il se tient loin de sa chère philologie.

D'ailleurs, sa santé se rétablira quand il lui aura fait ses adieux. Après les Commentaires de 1529, il se tourne vers une réflexion philosophique qui, en 1533, aboutira au De transitu hellenismi ad christianismum. Ce traité avait été précédé, en 1532, du De philologia où Budé note: “... cette maladie qui m'a accablé pendant presque vingt-huit ans" 34 . La formule ne peut qu'indiquer que le mal ne se manifeste plus. On en trouve la confirmation dans Louis Le Roy: “... il se trouva plein de vigueur à la fin de sa vie et il affichait une bien meilleure santé dans sa vieillesse qu'au milieu de son âge et son tempérament 
sec et sa vigueur lui auraient assuré plusieurs années encore si une maladie fortuite ne l'avait emporté"35.

Un dernier aspect de la maladie de Budé doit être mentionné: c'est l'insistance avec laquelle il répète que celle-ci a constitué un obstacle à des réalisations plus considérables. Nous avons dit déjà qu'il la présente comme cause de l'interruption des Annotations aux Pandectes et des Commentaires. Mais Il y revient également à maintes reprises dans des contextes plus généraux. Dans ces cas, la maladie est généralement associée à sa situation d'autodidacte ${ }^{36}$, pour expliquer la modestie de son savoir. Voici un passage caractéristique: "Le destin et les circonstances m'ont désavantagé, privé que je suis de livres d'abord, de maîtres ensuite et, enfin, de compagnons d'études—c'est là le pire. Je passe sous silence les fréquentes interruptions de mon travail dues aux médecins qui me menaçaient de trépas si je ne modifiais incontinent mon régime de vie et ne renonçais pas aux livres" ${ }^{\prime 37}$. Ces remarques s'accompagnent le plus souvent d'un appel à l'indulgence pour la modestie de ses réalisations.

C'est ce que nous savons de la maladie de Budé. Laissons à plus compétent le soin d'en percer la nature ${ }^{38}$ et voyons la lettre à Guillaume Cop.

\section{G. BUDAEUS G. COPPO S.}

P. 1 Dudum cum me inuiseris aegrotantem: eo eram corporis animique / habitu: ut mihi tussim tandem tabificam metuendam prae me / ferrem. postera tui aduentus nocteusque eo inualuit morbus ut / perpeti eademque

5 acerrima tussi nullo ferme screatu uexatus / conuulsusque: matutino etiam nausea ad deliquium/ ferme animi exagitatus: aut exanimationem potius: cum grauissimum /atrocissimumque quoddam exemplum numen (ut ita dicam) ipsum / tussis editurum in me exputarem:

10 repente intra biduum / grassatoria illa mali atrocitas concidit elanguitque, ita/ demum screatu iam facili ac purulento recreatus: defun/ctum me pernicie haud dubia spe ferebam. quoad (ex / insomnia arbitror diuturna) in gravedinem delapsum, aut / etiam relapsum cum ueterno quodam me sensi: hoc genere dies /

P. 2 aliquot marcescens: cum mihi non iam homo: sed ursus:// aut $\tau \hat{\omega} v$

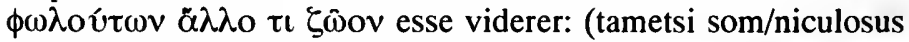
magis dormitatorque etiam et oscitator, quam tenore ullo / memorabili: aut gravitate somni oppressus)

5 cumque mihi displicens: / nulli iam $\phi i \lambda \circ \psi v \chi i \alpha_{\varsigma}$ incolumi retinaculo:

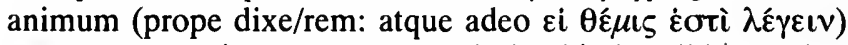
apocarteresmo impetu caeco / animi subinde mihi consciscerem: $\lambda$ óyov

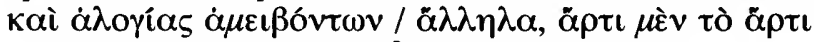

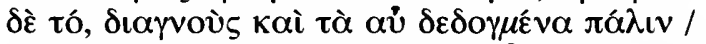

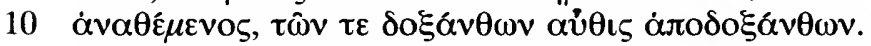
Cum igitur / huiusmodi fere essem mortis quidem securus: morbi uero / 
anxius: in universum autem nulla lucis cupiditate affectus: / ibi vero consilii tui memor: clystere prius usus $\pi v ́ \varepsilon \lambda o v \theta \varepsilon \rho / \mu \eta \dot{v}$, id est solium uaporatum ingressus: hemisphaerico

15 machi/namento capiti supposito, quo super solium eminebam: ut ea / quoque parte ex animi tui meique sententia uaporem excipe/rem: cum diu ac multum (tamen citra lassescen/tiam) desudassem: eo die toto clausum me continui, modo / aliquantisper commodiorem aut commodiusculam

20 ualetudinem / adeptus: iamque blandiende profectu

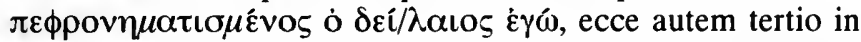
aliud; atque inde in aliud / malum eiuscemodi generis incido: nunc in oculos: nunc / in gingiuas: nonnunquam decumbente in ventriculum / morbo: interdum etiam in omnes simul partes:

25 ut mihi / [veni] rem ad triarios rediisse iure dicere possim, praesertim septen/ni iam ferme pernicie confecto: id quod ex me coram audisti / Cum interim nihil non expertus, ne morbi quidem cau/sam deprehendere

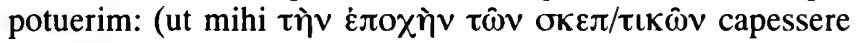
lubeat: et in causam pyrrhoniorum

30 incumbere)/ duntaxat absque te foret: qui mihi a fervore cerebri metuendum esse / admonuisti: quod tantopere miser confouendum esse rebar: / quamquam iam pridem iecinoris praecalidi uitium in causa esse

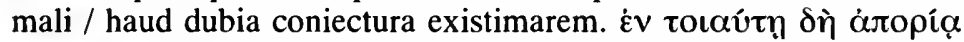

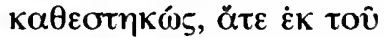

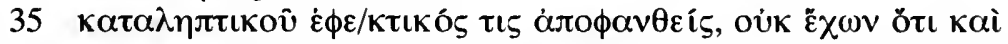

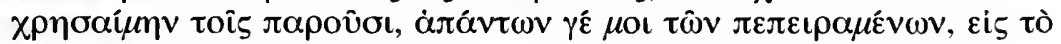

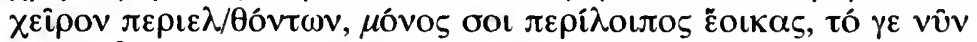

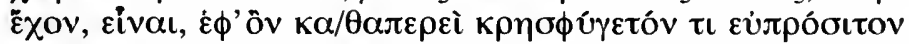

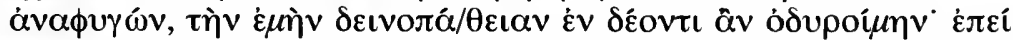

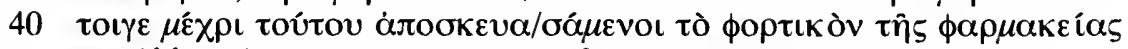

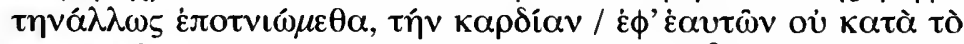

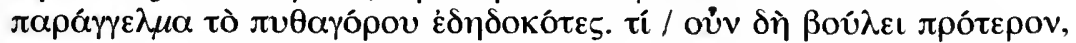

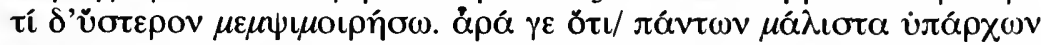

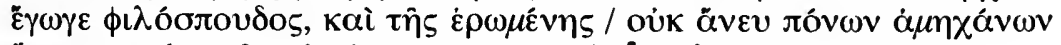

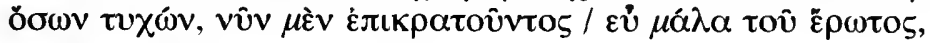

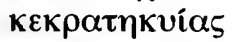

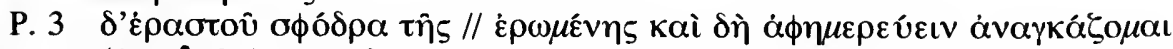

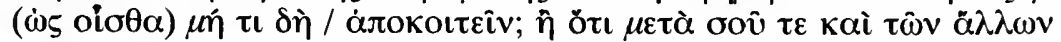

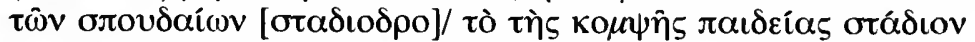

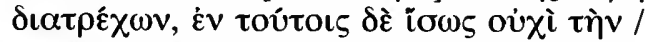

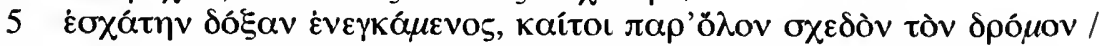

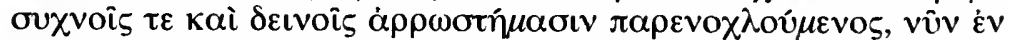

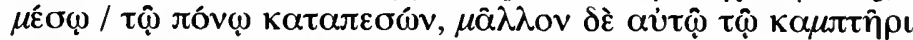

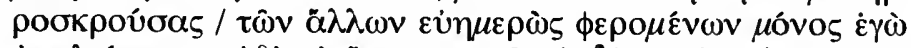

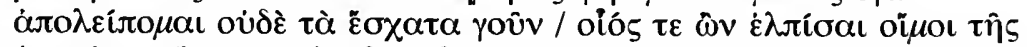

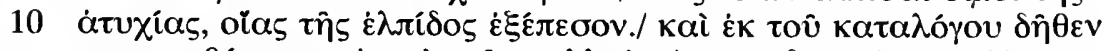

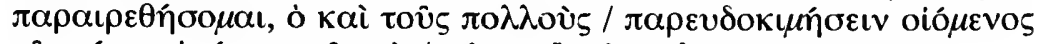

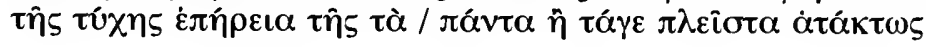




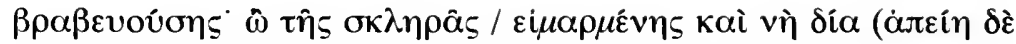

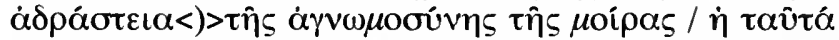

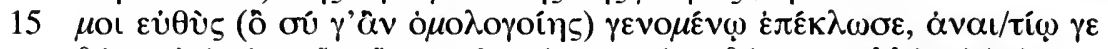

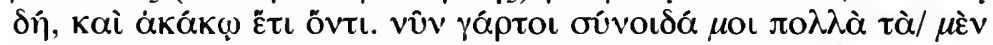

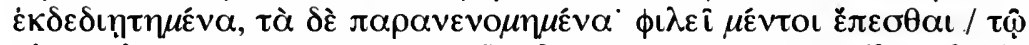

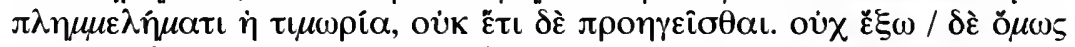

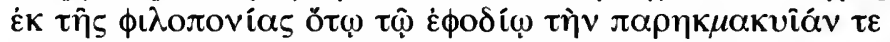

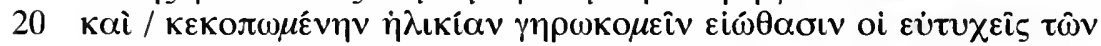

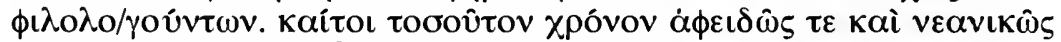

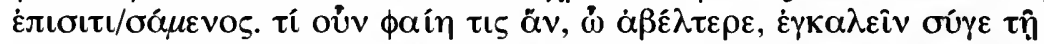

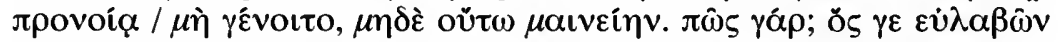

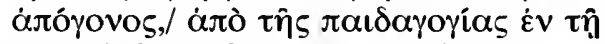

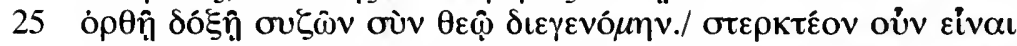

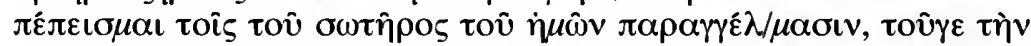

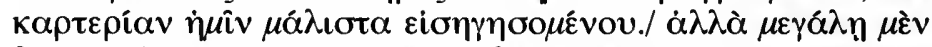

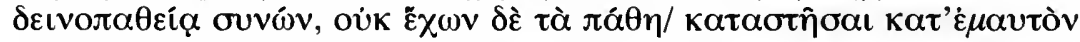

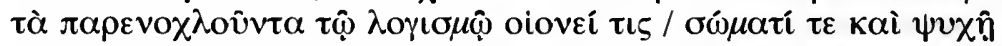

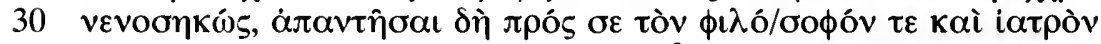

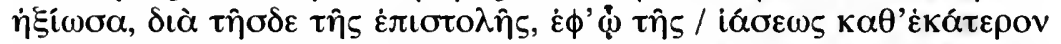

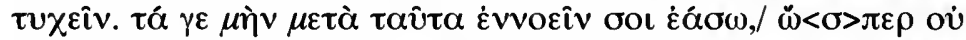

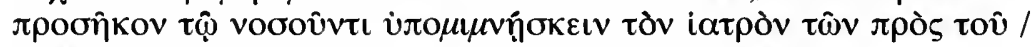

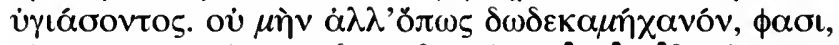

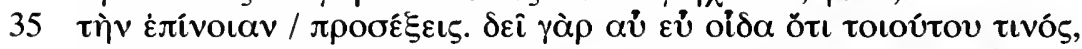

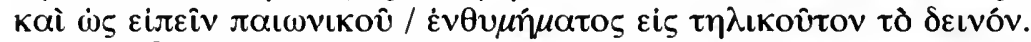

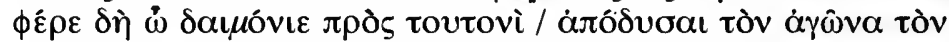

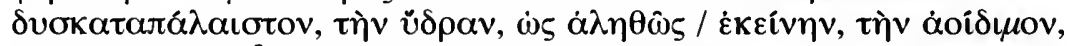

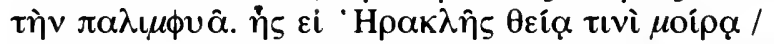

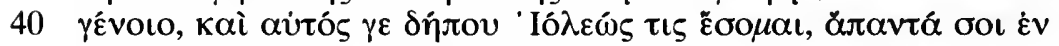

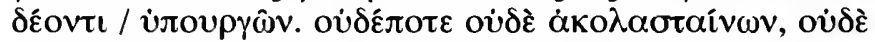

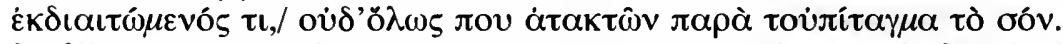

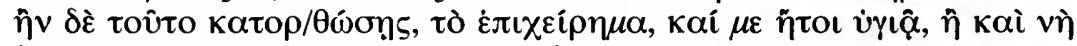

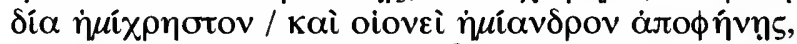

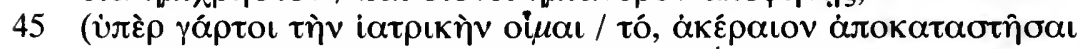

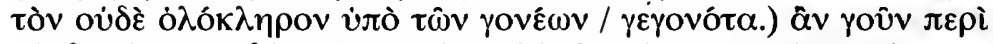

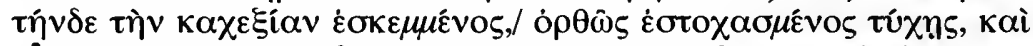

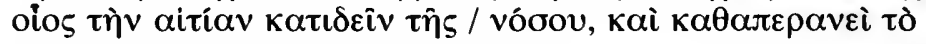

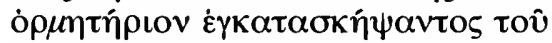

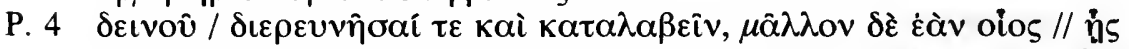

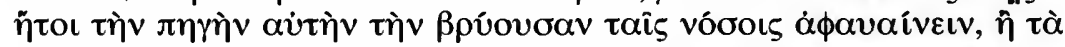

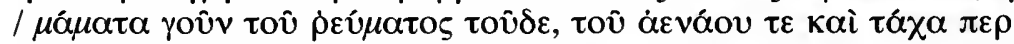

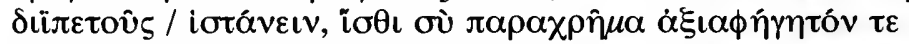

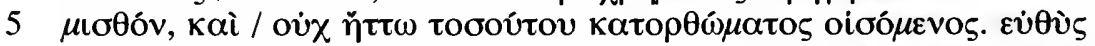

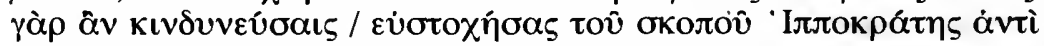

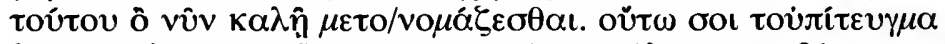

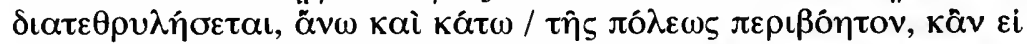

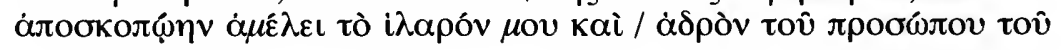




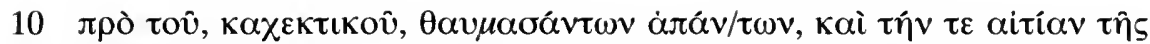

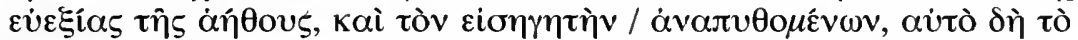

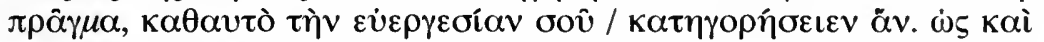

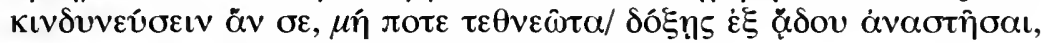

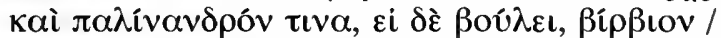

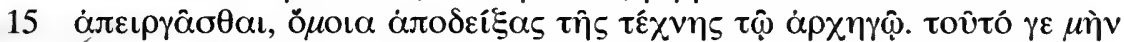

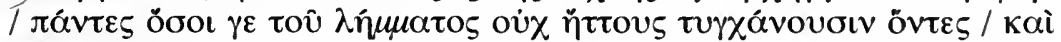

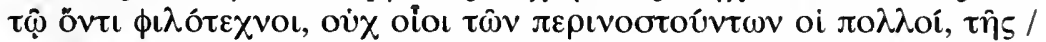

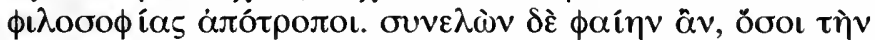

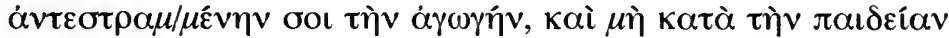

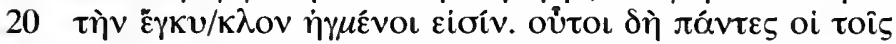

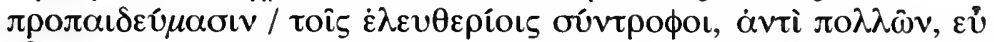

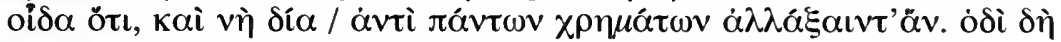

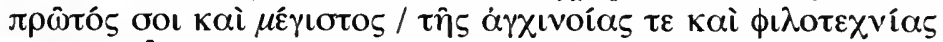

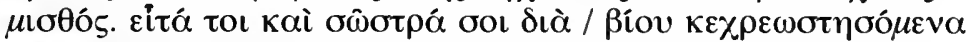

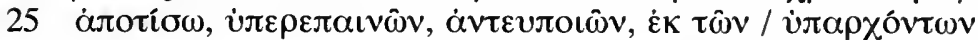

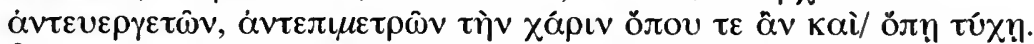

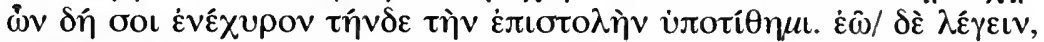

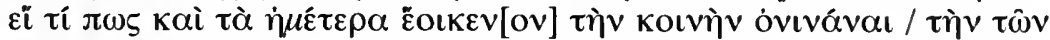

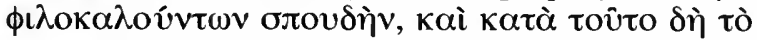

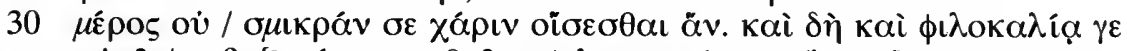

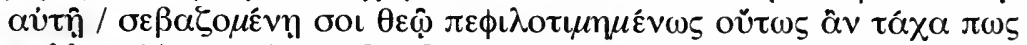

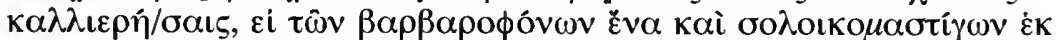

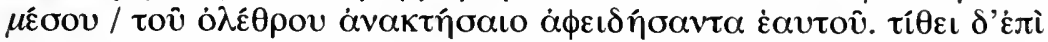

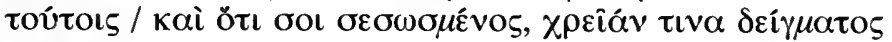

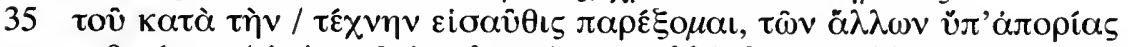

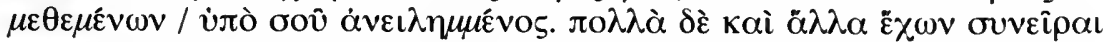

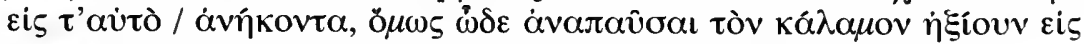

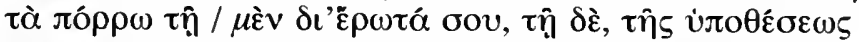

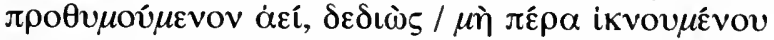

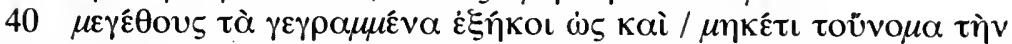

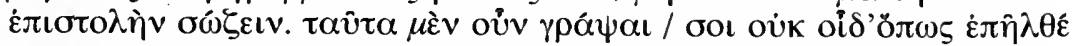

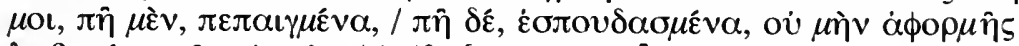

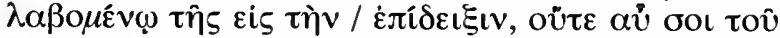

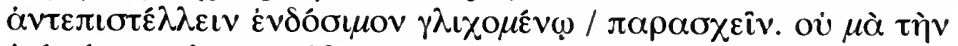

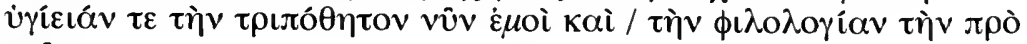

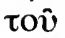

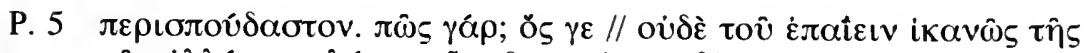

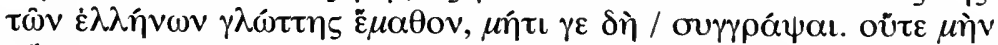

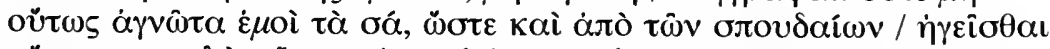

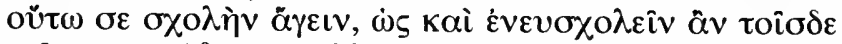

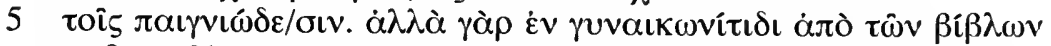

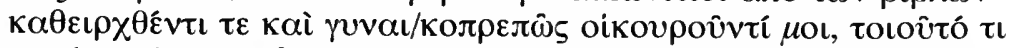

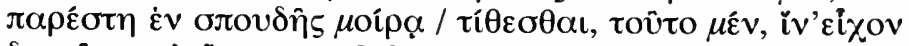

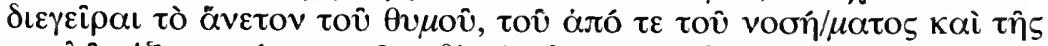

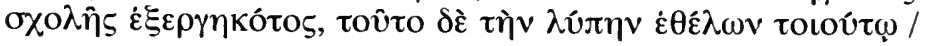




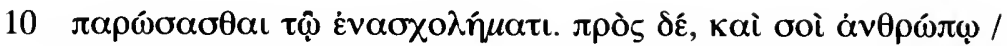

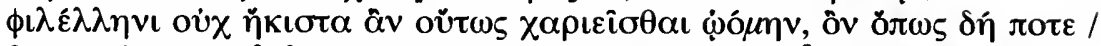

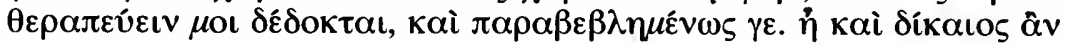

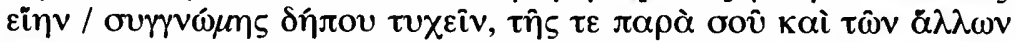

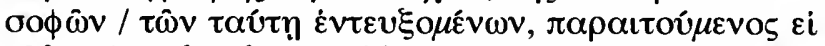

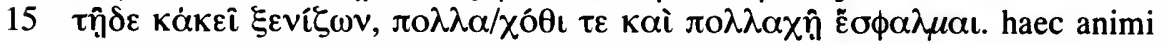
causa ad te scripsi: ac cum morbi / ludificandi gratia: morbidaeque molestiae per avocamentum animi transmittendae / tum vero abigendi cubicularis taedii. interim dum spatium recreandae / valetudini praebeo vel potius dum alieno

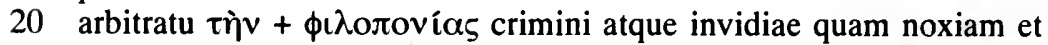

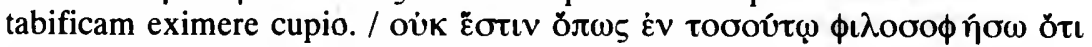

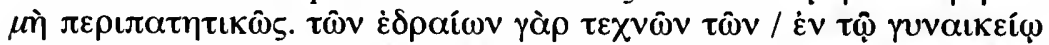

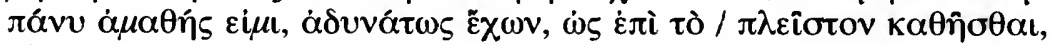

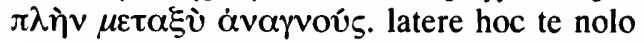

$25 \mathrm{me} / \mathrm{ex}$ quo sane epistolam scribere adorsus sum: interdiu quidem certe

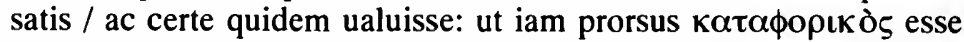

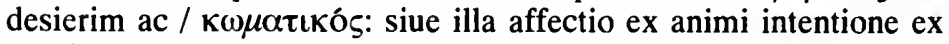
operis oblectamen/to tantisper laxamentum mihi praebuit: siue morbi materia penitus / aut

30 consumpta aut discussa est. Ceterum quidem, ut nonnulla ex / parte ludicram hanc epistolam, veluti seriosam habeas: quo mihi / aliquando

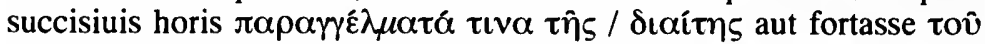

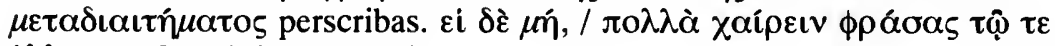

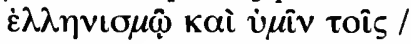

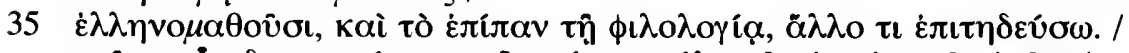

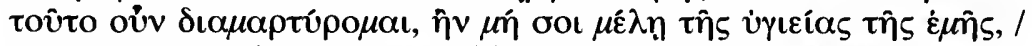

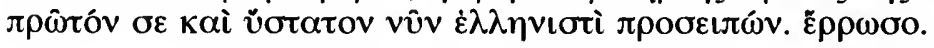

\section{CORRECTIONS}

En plus de ses abondantes ratures, le texte semble conserver quelques erreurs. L'absence fréquente de l'iota souscrit et les fautes d'accentuation ne sont pas signalées.

[ ... ]: delendum

$<>$ : addendum

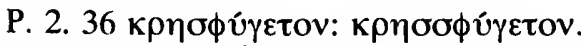

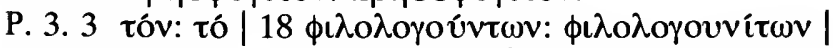

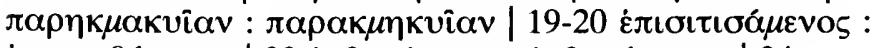

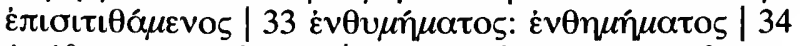

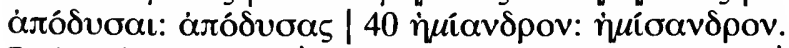

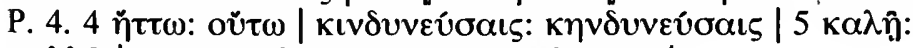

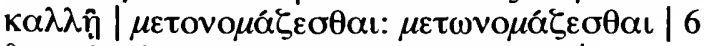

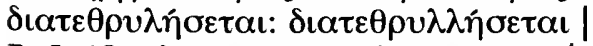

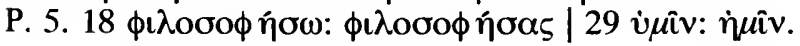




\section{GUILLAUME BUDÉ À GUILLAUME COP, SALUT!}

Quand tu m'as récemment visité au cours de ma maladie, j'étais de corps et d'âme dans un état tel que je présentais une toux dont on pouvait craindre qu'elle ne m'emporte. La nuit suivant ton arrivée, le mal a pris une ampleur telle que j'ai été péniblement secoué par une toux violente, ininterrompue et toujours égale, pratiquement sans aucune expectoration et que, le matin venu, j'ai finalement subi les assauts d'une nausée propre à me faire défaillir, ou plutôt à me faire rendre l'âme. Et au moment où je m'attendais à ce que la toux en personne, pour ainsi dire, montre en moi un modèle d'une gravité et d'une cruauté extrêmes, soudain, en deux jours, l'acuité tenace de ce mal est tombée et s'est évanouie. Finalement, soulagé par des expectorations désormais aisées et purulentes, c'est avec une confiance sans réserve que je me considérais à l'abri du danger--jusqu'au moment où (à cause, je pense, de longues insomnies) je me suis senti tombé ou, enfin, retombé dans une lourdeur accompagnée de somnolence.

J'ai langui dans cet état pendant quelques jours. Alors que j'avais le sentiment de n'être plus un humain, mais un ours ou quelque autre animal dans sa tanière (bien que je fusse engourdi et même somnolent et alangui plutôt que vaincu par un sommeil d'une durée ou d'une profondeur notables), alors aussi que, las de moi-même chez qui ne restait intacte aucune attache à la vie, j'allais céder (pourrais-je pratiquement dire, si toutefois il est permis de dire telle chose) à une pulsion aveugle de l'âme et décider de mon sort en me laissant mourir de faim-raison et déraison alternaient, c'était tantôt ceci, tantôt cela, je prenais une décision sur laquelle je revenais, ce qui avait paru opportun paraissait désormais inopportun-alors donc, dis-je, que je me trouvais dans cet état, à l'abri de la mort mais inquiet de la maladie et, en somme, sans aucun désir de vivre, je me suis souvenu de ton conseil: après le recours à un clystère, je me suis plongé dans un bain chaud, avec sous la tête un appareil hémisphérique pour me maintenir au-dessus du bain de sorte que la tête aussi reçoive tout plein de chaleur.

Après une sudation longue et abondante (sans toutefois me rendre à l'épuisement), j'ai gardé la chambre toute la journée, éprouvant pendant quelques temps une amélioration, du moins une certaine amélioration, à mon état. Tout fier, pauvre moi, d'un progrès encourageant, voilà que le troisième jour je me mets à tomber d'un mal à l'autre selon l'enchaînement suivant: le mal allait tantôt dans les yeux, tantôt dans les gencives, parfois il descendait dans le ventre et même à l'occasion partout en même temps. Si bien que je serais justifié de dire 
que j'en étais réduit à mes ultimes réserves, surtout qu'il y a bien sept ans déjà que je suis atteint de ce mal, comme tu me l'as entendu dire de vive voix.

Et pourtant, après avoir tout essayé, je n'ai pas été capable de trouver la cause du mal (si bien que je me suis laissé aller à embrasser la retenue des Sceptiques et à adopter la cause des Pyrrhonien ${ }^{39}$ ), du moins si je ne tiens pas compte de toi, qui m'as prévenu du danger pour moi d'un réchauffement du cerveau, que moi-quel malheureux!-je croyais devoir également réchauffer. Il y avait d'ailleurs longtemps que j'attribuais hors de tout doute la cause du mal à l'anomalie d'un excès de chaleur au foie.

Placé dans un tel embarras, vu le scepticisme auquel m'a mené mon analyse, ne sachant que faire dans cette situation, puisque tous mes efforts ont empiré le mal, j'ai l'impression de n'avoir plus que toi, du moins pour le moment, vers qui me tourner comme vers un refuge accueillant, pour me plaindre utilement de mes souffrances. Car jusqu'ici, l'intestin vidé, c'est en vain que j'ai eu recours aux désagréments des purgatifs-le coeur maintenant rongé, mais pas dans le sens du précepte de Pythagore ${ }^{40}$.

A ton avis, donc, à quoi m'en prendre d'abord, à quoi ensuite? Est-ce parce que moi, ardent entre tous, ne profitant de mon amante qu'au prix d'efforts pratiquement impossibles, parce que, donc, maintenant que cet amour est absolument souverain et que l'amante a pleine domination sur l'amant, me voici contraint de m'en éloigner pendant le jour, comme tu le sais, et, à plus forte raison, de délaisser sa couche? Ou encore, parce que, alors que je parcourais avec toi et les autres enthousiastes le stade de la belle éducationmais sans peut-être y avoir atteint les sommets de la renommée et en butte tout au long de ma course à de terribles et fréquentes défaillances-parce que, dis-je, maintenant tombé en plein élan, ou plutôt brisé contre la borne pendant que les autres poursuivaient avec bonheur, me voici laissé seul en arrière, sans espoir possible d'atteindre le but ${ }^{41}$. Hélas! quel revers! De quel espoir j'ai été frustré! Et sans doute serai-je rayé du palmarès, moi qui croyais m'élever au-dessus de la masse: perfidie de la fortune qui mène tout, ou presque, dans le désordre! $O$ rigueur du sort et, par Zeus (loin de moi Adrastée ${ }^{42}$ ), ô aveuglement du destin qui (ce dont tu conviendrais) m'a ménagé tout cela dès ma naissance, alors que j'étais encore ignorant de l'erreur et du mal. Bien sûr, je suis conscient qu'aujourd'hui mes errements et mes fautes abondent. Mais le châtiment suit habituellement l'offense, il ne la précède pas.

De mon attachement au travail, je ne tirerai pourtant pas le viatique par lequel ceux qui pratiquent la philologie ${ }^{43}$ avec bonheur ont l'habitude d'assurer la maturité, le déclin de leur âge ${ }^{44}$. C'est pourtant sans ménagement, avec vaillance, que j'ai peiné à accumuler mes provisions. "Mais, quoi!" 
dira-t-on, "pauvre sot, ne va pas t'en prendre à la Providence!" Dieu me garde de telle folie! Et comment donc? Moi qui suis né de parents pieux, depuis mon enfance je n'ai cessé de vivre dans la droite voie près de Dieu. Je suis donc convaincu qu'il faut s'attacher aux préceptes de notre Sauveur, lui qui certes nous insufflera la constance.

Mais, accablé par une maladie terrible, incapable par moi-même de mettre fin aux souffrances qui entravent mes facultés, comme un dont le corps et l'âme sont attaqués, j'ai voulu par cette lettre m'adresser à toi, philosophe et médecin, dans l'espoir de trouver la guérison tant du corps que de l'âme. Je te laisserai réfléchir à ce qu'il faut faire, puisqu'il ne revient pas au malade de dicter le traitement au médecin. Néanmoins, veille à porter à la question une attention aux mille astuces, comme on dit, car, je le sais bien, il faut un homme de cette sorte et, pour ainsi dire, doué de la perspicacité de Paeon ${ }^{45}$, face à un tel mal. Allons, mon brave, retire tes vêtements pour affronter cet adversaire coriace, l'Hydre en vérité, si célèbre, sans cesse renaissante. Si par un plan divin, tu devenais pour elle un Héraclès ${ }^{46}$, je serai moi-même un Iolaos, qui t'assistera en tout opportunément, qui jamais ne se permettra de dérèglements, jamais le moindre écart de régime, jamais, bref, la moindre dérogation à tes ordres.

Si tu mènes à bien cette entreprise et que, ou bien tu me guérisses, ou bien, par Zeus, tu me rendes une demi-utilité, tu fasses de moi en quelque sorte un demi-homme (car cela dépasse la médecine, je pense, que de donner l'intégrité à celui que ses parents n'ont pas engendré complet), si donc, après un examen de cette affection, tu arrives à un juste diagnostic et parviens à identifier la cause de la maladie et, en quelque sorte, à découvrir, à surprendre le repaire du mal qui m'a frappé, ou plutôt, si tu parviens à sécher la source elle-même d'où jaillissent les maladies, ou du moins à tarir l'aliment de ce flot ininterrompu et peut-être bien d'origine céleste, ${ }^{47}$ alors sache qu'à l'instant tu toucheras un salaire digne de mémoire et non en reste d'un tel succès. Car, sur-le-champ, tu risquerais, le but atteint, de prendre le nom d'Hippocrate ${ }^{48}$ à la place de celui que tu portes maintenant, tant on clamera ta réussite, célébrée d'un bout à l'autre de la ville. Et si l'on m'examinaittous s'étonnant de la gaieté et de la santé de mon visage auparavant maladif et s'informant de la cause de cette vigueur inaccoutumée et de son auteur-, d'elle-même la chose révélerait ton bienfait. De la sorte, tu aurais des chances de passer pour avoir ramené de l'Hadès un défunt et, si tu veux, de l'avoir rétabli dans la condition d'homme avec une seconde vie ${ }^{49}$, faisant preuve d'un art comparable à celui du Créateur.

Voilà certes chose que tous ceux qui, insensibles à l'appât du gain, ont la véritable passion de l'art-à la différence de ces amateurs d'artifices, de la 
masse étrangère à la philosophie, en somme, dirais-je, de ces gens dont la formation s'oppose à la tienne et qui n'ont pas suivi la voie de l'encyclopédie ${ }^{50}$ —chose donc que tous ceux qui ont été nourris des éléments d'une éducation libérale échangeraient, j'en suis convaincu, contre bien des richesses et même, par Zeus, contre toutes les richesses. Et ce sera là pour toi la première et la plus appréciable rémunération de ta perspicacité et de ton amour de l'art. Ensuite, la rétribution à laquelle tu auras droit pour toute ta vie, je te la verserai en te couvrant d'éloges, en te comblant à ton tour de bienfaits, en employant mes ressources à te rendre tes bons procédés, en te témoignant en toute circonstance et par tout moyen qui s'offrira une reconnaissance sans borne. Je t'en donne comme gage la présente lettre. Je passe sous silence que, si j'ai quelque apparence d'avoir soutenu l'ardeur commune des adeptes des belles lettres, en cela aussi ce n'est pas une mince gratification que tu récolterais. Et précisément, cet amour de l'art pour lequel ta vénération rivalise avec celle de Dieu, peut-être bien la plus belle offrande que tu pourrais lui faire serait-elle d'arracher du sein de la ruine un pourfendeur des barbares, un châtieur des béotiens ${ }^{51}$, lui qui a agi au mépris de son propre bien. Ajoute à cela que, sauvé par toi, je servirai par la suite à illustrer la puissance de ton art, puisque tu m'auras recueilli quand les autres à bout de ressources m'avaient abandonné.

Bien que j'aie matière à m'étendre encore longuement sur ce sujet, j'ai jugé bon mettre ici un frein à ma plume toujours poussée de l'avant par mon affection pour toi et par le sujet lui-même. Je crains que, si je m'étends davantage, ce texte tourne de telle sorte que ma lettre ne mérite plus son nom $^{52}$. En fait, je ne sais trop comment il m'est venu à l'esprit de t'écrire tout cela -en partie plaisanteries et en partie propos sérieux. Je n'en ai certes pas trouvé l'inspiration dans le goût de l'étalage, non plus que dans le désir de te fournir une occasion de m'écrire à ton tour. Non, par la Santé ${ }^{53}$ aujourd'hui trois fois désirée et par la Philologie hier si affectionnée! Comment donc l'aurais-je pu? moi qui n'ai pas suffisamment appris à comprendre la langue des Grecs—et encore moins à l'écrire—et qui n'ignore pas non plus ta situation au point de croire que tes obligations te laissent assez de loisir pour que tu puisses t'adonner à ces amusements.

Mais, comme je suis confiné aux appartements des femmes, loin de mes livres et oisif comme les femmes, il m'est venu à l'idée de remplacer l'étude par ce genre d'occupation: d'un côté, je pouvais de la sorte secouer l'engourdissement de mon esprit endormi par la maladie et le désoeuvrement et, de l'autre, chasser l'ennui par ce passe-temps! En outre, philhellène comme tu l'es, j'estimais que ce n'était pas le moindre moyen de t'être agréable, à toi 
envers qui il me semble justifié d'avoir tout l'empressement possible - et avec un dévouement aveúgle. Je serais certes digne de votre indulgence, à toi et aux autres savants qui verront cette lettre, si je reconnais que, ballotté ici et là,j'ai trébuché en maints endroits et à maintes reprises.

C'est pour le plaisir que je t'ai écrit cette lettre, tant pour tromper le mal et en oublier les désagréments en distrayant mon âme que pour chasser l'ennui de ma chambre. Alors que je donne à ma santé le temps de se refaire, ou plutôt que, sur l'avis d'autrui, je cherche à réparer ${ }^{54}$ le tort causé par ma passion du travail, il ne m'est pas possible, pendant ce temps, de m'adonner à l'étude, sauf en marchant. Je suis en effet tout à fait étranger aux travaux sédentaires des appartements des femmes, étant incapable de rester presque toujours assis, si ce n'est pour lire.

Je ne veux pas te cacher que, depuis que j'ai commencé à écrire cette lettre, ma santé a pris assez et même beaucoup de mieux, de sorte que j'ai cessé de me sentir profondément somnolent et léthargique: ou bien l'état créé par la tension de l'esprit due au plaisir de la tâche m'a procuré une certaine détente, ou bien la matière de la maladie est totalement épuisée ou dissoute. Pour le reste, dans cette lettre écrite pour l'agrément, puisses-tu voir un peu de sérieux, de sorte que tu profites de moments libres pour me coucher par écrit quelque ordonnance sur mon régime, ou peut-être sur un changement de régime. Sinon, je ferai bien des saluts à l'hellénisme, à vous, adeptes du grec, et surtout à la philologie, puis je m'adonnerai à autre chose. Mais, crois-en ma parole, si tu ne te soucies pas de ma santé, c'est aujourd'hui la première et la dernière fois que je m'adresse à toi en grec. Au revoir.

\section{Université de Montréal}

\section{Notes}

1. Voir le $\mathrm{n}^{0} 102$ de ce Catalogue, préparé par M. Gasnault, conservateur au Département des Manuscrits, et Mme Verrin-Forrer, conservatrice au Département des Imprimés (Paris 1968).

2. Ernest Wickersheimer a consacré à Guillaume Cop (c. 1466-1532) une substantielle notice biographique dans le Bulletin de la société française d'histoire de la médecine (1913, XII, pp. 336-342). Cette notice est reprise en substance dans son Dictionnaire biographique des médecins en France au Moyen-Age (Paris, Droz, 1935). Rappelons ici brièvement que ce médecin humaniste originaire de Bâle était lié d'amitié avec les plus grandes figures de son époque, dont Lefèvre d'Etapes, Jean Lascaris et Jérôme Aléandre (tous deux lui avaient enseigné le grec), Erasme, qu'il eut l'occasion de soigner et qui lui dédiera son De Senectute. C'est d'ailleurs sans doute en vertu de cette amitié que François ler $^{\text {er }}$ chargera Cop de faire venir Erasme en France en 1517 pour diriger le collège projeté. A partir de 1513 , peut-être de 1512 , il sera le médecin de Louis

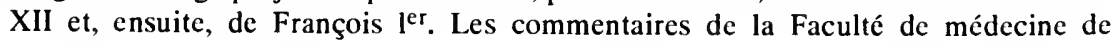


l'Université de Paris (publiés par E. Wickersheimer, Paris, Imprimerie nationale, 1915) permettent de suivre sa trace dans cette institution, d’abord comme étudiant, puis comme professeur. Guillaume Cop a publié des traductions, maintes fois rééditées, d'Hippocrate, de Galien, et de Paul d'Egine.

3. Lignes 23-24 à la page 2 de la copie.

4. Emile Legrand, Bibliographie hellénique Il, Maisonneuve et Larose, 1962, pp. 332-333 (Réimpression de l'édition de 1885).

5. Opera omnia G. Budaei I, p. 378, Bâle 1557 (réimpression Gregg, Farnborough, 1966 et 1969).

6. Ibid., p. 251. Curieusement, trois semaines plus tard, le 21 février, Budé écrira à Longueil: “. . . le mal incrusté en moi que je traîne pour une quatorzième année déjà sans pouvoir m'en défaire."(Ibid. p. 273)

7. Budé est moins précis dans une lettre à Nicolas de la Chesnaye en date du 5 juillet 1519: "Rien ne m'est plus à coeur que de recouvrer la bonne santé que j'ai perdue il y a quatorze ans et plus." (ibid. I, p. 265). De même, dans le De philologia, Budé dit avoir souffert pendant "presque vingt-huit ans"(ibid., p. 95). Ce qui, compte tenu du presque, n'exclut pas absolument l'année 1505. Il est encore plus vague dans le De asse - dont la préface est datée du 15 mars 1514 - où il fait remonter le début de sa maladie à "plus de sept ans" (ibid. II, p. 306). Restent deux références plus problématiques. D'abord, une lettre à Nicole Bérault, datée du 25 mars 1511 dans les Epistolae de 1520, où Budé écrit: "Voici maintenant quatre ans que je livre un combat incertain contre un mal absolument terrible." (ibid. I, p. 260). Ensuite, dans la lettre à Tunstall du 19 mai 1517, il estime sa maladie "d'autant plus pénible qu'elle est responsable des calomnies répandues depuis quinze ans aussi bien par les médecins que par mon entourage." (ibid., I, p. 362). Dans ce dernier cas, la maladie remonterait à 1503 et, dans l'autre, à 1507 . Pour diverses raisons, toutefois, il me semble légitime de se demander s'il n'y aurait pas eu erreur de typographie dans la date de la lettre à Béreault. Notamment, sans compter qu'une date aussi tardive est contredite par tous les autres documents, elle suppose que Budé a connu une crise qui, éclatée pendant la rédaction des Annotations aux Pandectes terminées en novembre 1508, durait toujours en mars 1511, puisque Budé note dans cette lettre: “cette mauvaise santé ne m'a pour ainsi dire pas laissé une heure depuis la parution de mon livre jusqu'à aujourd'hui. Malgré ces contradictions, je tiens comme probable qu'il faille placer en 1505 le début de la maladie de Bubé et, conséquemment,accepter de la même façon la date de $15: 12$ pour la lettre à Guillaume Cop.

8. Opera Omnia I, p. 329 B.

9. Lignes 11-12, page 5 de la copie.

10. Opera Omnia I, pp. 402-405; 356-364; 406-409.

11. Ibid. IV, les deux pages suivant la colonne 1560 .

12. Voir notamment les lettres à Tunstall et à Longueil mentionnées plus haut.

13. Voir H. Brabant: Erasme, humaniste dolent, Presses académiques européennes, 1971. Cette étude est reprise en substance dans: "Erasme, ses maladies et ses médecins", Colloquia Erasmiana Turonensis I, Paris, Vrin, 1971, pp. 539-568.

14. Librorum emacissimus, dit Budé dans la lettre à Tunstall (ibid. I, p. 362).

15. De philologia, dans Opera Omnia I, p. 35. 
16. Henri Omont, "Notice sur les collections de manuscrits de Jean et Guillaume Budé", Bulletin de la Société d'Histoire de Paris XII, 1885, pp. 100-113.

17. E. Legrand, op. cit., tome II, pp.332-333. La Thérapeutique de Galien avait été publiée par Nicolas Vlastos à Venise en 1500. Il est intéressant de noter que la faculté de médecine de Paris n'achètera les oeuvres de Galien qu'en 1526 et celles d'Hippocrate qu'en 1527 (voir Ernest Wickersheimer, La médecine et les médecins en France à l'époque de la Renaissance, Paris 1905, p. 42 (Slatkine Reprints, 1970).

18. Voir lettres à Thomas Linacre du 10 juillet 1517 (Opera omnia I, pp. 248-250) et du 9 septembre 1518 (ibid., p. 250), à Erasme du 7 juillet 1516 (ibid., pp. 366-368) et à Lupset du 31 juillet 1517 (The Complete Works of Thomas More, ed. E. Surtz et J. H. Hexter, Yale Univ. Press, IV, pp. 5-14) et du printemps 1519 (Galeni Methodus Medendi, Paris 1519 r\$du feuillet 2).

19. Voir lettre du 31 juillet 1517 à Thomas Lupset citée dans la note précédente, p. 14. Jean Ruel (1479-1537) est l'un des deux premiers titulaires nommés aux chaires de médecine créées en 1505. Médecin de François I I r Ruel sera nommé chanoine à Notre-Dame de Paris vers la fin de sa vie. Henri Estienne publia de lui en 1516 une traduction de Dioscoride souvent reprise. En 1536, Ruel donna chez Simon de Colines son De natura stirpium dont le seizième siècle a connu quatre réimpressions.

20. Il faut toutefois signaler un passage d'une lettre à Erasme en date du 14 décembre 1522 (Opera Omnia I, p. 378). Venant d'être nommé Maître des Requêtes, Budé écrit: “Mais je n'ai pu paraître dans cette dignité à cause d'un mal nouveau-né, rejeton de cette ancienne maladie si familière. Cette année, une humeur plus âcre et mélangée de bile s'est mise à descendre aux intestins en y provoquant des coliques accompagnées de vomissements violents, sibien que personne n'ose me promettre la moindre prolongation de ma vie". Dans une autre lettre à Erasme du début de 1523 (ibid., p. 379), Budé rappelle cette maladie, qu'il distingue encore explicitement de son mal habituel: "C'est de la tête jadis, de l'intestin récemment et de partout où loge la vie que je souffre à en défaillir."

21. Il faut considérer que la maladie de Budé a effectivement duré plus de vingt ans, puisque, commencée en 1505 , elle a connu une de ses pires manifestations lors de la préparation des Commentaires terminés en 1529. Cette crise, particulièrement grave, a vraisemblablement pu être la dernière. Budé avait alors 61 ans.

22. "Tousser bruyamment" repose sur une interprétation de "tumultuose" dans l'expression "jugulum tumultuose appetens" par laquelle Louis Le Roy qualifie la maladie. Je crois que cette interprétation ne fait pas de doute. Outre que "avec bruit" est le sens premier de "tumultuose," il est pratiquement impossible d'expliquer les tapes dans le dos ("humeros feriendo") dont il sera question plus loin si Budé ne toussait pas. Je dois toutefois noter que, à ma connaissance, ceux qui ont auparavant examiné ce passage ne l'ont pas compris ainsi. Eugène de Budé (Vie de Guillaume Budé, Paris 1884, p. 23), Louis Delaruelle (Guillaume Budé. les origines, les débuts, les idées maîtresses, Paris 1907, p. 84), David O. Mc Neil (Guillaume Budé and Humanism, Genève 1975, p. 7) et L.F. Flutre (“L'étrange maladie de Guillaume Budé”, Aesculape, janvier 1938, pp. 3-6), ne parlent que d'un mal de gorge, pas de toux. McNeil estime que les tapes dans le dos avaient comme but d'amener Budé à se retourner pendant son sommeil pour éviter la suffocation, ce qui semble peu vraisemblable.

23. Faut-il ajouter foi à ce détail? Que la Vita ait été publiée l'année même de la mort de Budé et, en plus, soit dédiée à Guillaume Poyet, intime de Budé, rend difficile une 


\section{4 / Renaissance and Reformation}

invention susceptible d'être aussitôt démentie. Eugène de Budé (op. cit, p. 24) rapporte que la cautérisation du dessus de la tête était pratiquée pour certaines maladies.

24. La Vita Budaei de Le Roy figure au début du tome 1 des Opera Omnia. Le passage cité commence au bas de la deuxième page du feuillet EE4.

25. Voir note 21.

26. Lettre à G. de Brie, 2 sept. 1521 (Opera Omnia 1, p. 413)

27. 15 octobre 1518, Opera Omnia I, p. 407. On notera avec intérêt que, en juin 1500, Erasme relate à Lord Mountjoy avoir écrit ses premiers adages pendant une maladie, "en trompant pendant ce temps mon médecin, qui me menaçait d'un châtiment si je touchais seulement à un livre." (La correspondance d'Erasme 1, trad. Marie Delcourt, Bruxelles 1967, p. 264). Ce médecin était précisément Guillaume Cop.

28. Lettre à Tunstall du 10 juin 1517. Opera Omnia 1, p. 358.

29. Lettre-préface aux Annotations, feuillet a2, r\$.

30. Ibid., feuillet a3, v\$.

31. Opera Omnia IV, page suivant la col. 1560.

32. Voir la lettre à son frère Louis déjà mentionnée.

33. Lettre écrite à Pierrelatte, le 22 avril 1519, Opera Omnia I, p. 409.

34. (Ibid. I, p. 95: “... quae mihi annos fere duodetriginta infestior fuit.”

35. "Dans les grandes chaleurs de juillet, qui furent cette année-là les plus extrêmes de mémoire d'homme, le Roi s'était retiré sur la côte de Normandie pour fuir la chaleur et Budé, attaché à Poyet, l'avait suivi par ce même ciel si hostile. Soit à cause du vice de l'air, soit à cause de l'excès de chaleur qui brûlait alors tout, soit à cause de la faiblesse due à un âge avancé, il contracta une fièvre intense et continue dont il ne se remit pas. Malgré toutes leurs assurances et leurs promesses de guérison, les médecins ne purent jamais le convaincre que cette maladie n'allait pas mettre un terme à sa vie." (Louis Le Roy, op. cit., FF4 v\$). Le dernier détail entre en contradiction, au moins apparente, avec ce que Budé écrit à Poyet le 8 août pour prendre congé: "Je rejoindrai ta suite (...) dès que ma santé me le permettra. D'un même coup, en effet, j'ai confiance que, à moi, ma santé d'hier, à toi, ma personne seront rendues." (Lettre inédite, B.N. Mss, nov. acq. françaises 22338, F. 12).

36. Dès les premiers textes que nous avons de Budé (en 1503, dans la lettre à Germain de Ganay, Opera Omnia 1 p. 509, et dans la lettre à Pierre de Courthardy, ibid., p. 484), le désavantage d'avoir étudié seul est mis en relief. Budé y reviendra sans cesse, jusqu'à la postface aux Commentaires inclusivement.

37. Lettre à Longueuil du 15 oct. 1518 (Opera Omnia 1, p. 407).

38. Il pourrait être tentant de comprendre comme psychosomatique la maladie de Budé. Un collègue longtemps professeur de pathologie le suggère. La sensibilité de Budé à l'opinion qu'on se fait de lui peut nourrir cette hypothèse. Rappelons, parmi tant d'autres, ce passage d'une lettre du 21 avril 1527 à Erasme: “... depuis huit mois déjà il me pèse de secouer mes papiers (les brouillons des Commentaires) déjà couverts de poussière, même si je m'étais lancé dans ce travail avec détermination. Que ne m'est-il possible de les abandonner sans craindre le déshonneur!" (Opera omnia 1, p. 381). La maladie présente toujours une justification étanche. Mais sans doute est-il sage 
d'écouter les appels à la prudence de Léon-E. Halkin dans sa Psychohistoire et critique historique: le cas d'Erasme (Université de Liège. 1984.).

39. Le sentiment qu'il était impossible de connaître la vérité amenait les adeptes du scepticisme à suspendre leur jugement. Pyrrhon d'Elis (c. 360-275), dont on connaît la pensée seulement par ses disciples, a été le principal théoricien de cette école.

40. Mot cité par Plutarque (2.12e), qui pour en expliquer le sens rappelle les mots de Thétis à Achille: "Mon enfant, jusques à quand resteras-tu à gémir et à t'affliger, à te ronger le coeur?" (Il. 24, 128-129). Une scholie à ce passage de l'Illiade commente: "Pythagore exhorte à ne pas se ronger le coeur, c'est-à-dire à vivre sans chagrin et sans trouble."

41. Cette comparaison avec les compétitions du stade - autre lieu commun chez Budé sera particulièrement développée dans la lettre à Longueil du 15 octobre 1518 (Opera Omnia I, p. 406 ss.).

42. Adrastée avait d'abord été une épithète de Némésis, personnification de la vengeance divine qui frappait l'homme oublieux des limites propres à sa condition. L'exemple le plus célèbre est celui d'Agamemnon.

43. Budé définit la philologie comme suit: “... et s'appelle philologie: c'est-à-dire, désir et amour des bonnes lettres, et fervente inclination à l'estude des sciences, qui se nomment libérales, pour ce qu'elles requièrent l'homme de franche condition et estime, hors de servitude, cupidité, et ambition." (Institution du Prince, Arrivour 1547. Réimpression Gregg, Farnborough, 1966).

44. Plainte qui reviendra souvent. Budé rappellera à plusieurs reprises ses espoirs déçus quand il cherchera à obtenir des avantages du roi. Le plus bel exemple figure dans la lettre du 14 septembre 1521 à Jean Lascaris, dans laquelle Budé raconte comment il a été frustré d'une pension promise par François $1^{\mathrm{er}}$ (cf. Opera Omnia I, p. 424 s.).

45. Les poèmes homériques présentent Paeon comme un dieu guérisseur. Toutefois, par la suite, le nom apparaîtra en général comme simple éphitète d'Apollon.

46. Parmi les douze travaux d'Héraclès figure sa victoire sur l'Hydre des marais de Lerne, en Argolide. lolaos, qui était son cocher, l'assista dans cette entreprise.

47. Il est difficile de saisir la pensée de Budé, mais il s'agit très probablement d'une référence à l'influence des astres sur les maladies. Très répandues, ces croyances ont même retenu l'attention d'un médecin aussi rationaliste que Jean Fernel (cf. Léon Figard, Un médecin philosophe au XVIe siècle, Paris 1903, Slatkine Reprints, Genève

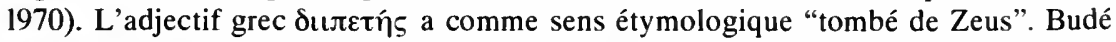
pourrait donc vouloir dire "envoyé par Dieu". Mais c'est très improbable. Dès l'époque

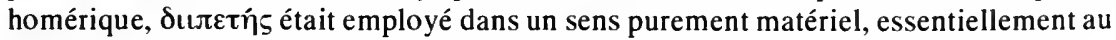
sujet de l'eau ou de la lumière qui tombent du ciel.

48. Dans une lettre à Jacques Wimpfeling en date du 21 septembre 1514 , Erasme appellera justement Cop "l'Hippocrate de notre temps" (La correspondance d'Erasme II, traduite par M.A. Nauwelaerts, Bruxelles 1974, p. 31).

49. Le texte donne $\beta i ́ \rho \beta ı v$, mot incompréhensible. L'hypothèse la plus satisfaisante à laquelle j'arrive est de lire díoßıov. Le mot serait alors un néologisme (ils sont

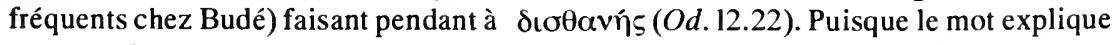
$\pi \alpha \lambda i ́ v \alpha v \delta \rho o v$, cette hypothèse est du moins satisfaisante pour le sens. 


\section{6 / Renaissance and Reformation}

50. Le mot n'a évidemment pas encore le sens qu'il prendra avec Diderot. Il s'agit ici essentiellement de la connaissance de l'Antiquité. Voir Marcel François, "Encyclopédie", Renaissance Quarterly XXIII, 1970, pp. 276-277.

51. Littéralement: "un fouetteur des Soloikoi" (du nom de la ville de Soloi en Cilicie), dont le mauvais parler est à l'origine du mot solécisme.

52. L'habitude qu'avait Budé de se laisser entraîner par son sujet a donné au De asse "quatre fois la taille prévue" (Opera Omnia II, p. 106). De même, dans les Commentaires, "l'enchaînement continuel des idćes allongeait la toile" (postface aux Commentaires, Opera Omnia IV, page suivant la col. 1560). On trouve des remarques analogues dans les Anotations (v.g. Opera Omnia III, p. 217 et 218).

53. Les Grecs personnifiaient la Santé dont ils faisaient une déesse.

54. Ce passage comporte manifestement une lacune. Le signe + se trouve dans le texte. 